\title{
Modelling and simulation of photocatalytic oxidation mechanism of chlorohalogenated substituted phenols in batch systems: Langmuir-Hinshelwood approach
}

\author{
Z. Khuzwayo, E.M.N Chirwa
}

Department of Chemical Engineering, Water Utilisation Division, University of Pretoria, Pretoria, 0002 South Africa. Tel.: +27793797619, E-mail: zack.khuzwayo@up.ac.za.

\begin{abstract}
This study investigated, modelled and simulated the influence of multi-chlorohalogenation in heterogeneous photocatalytic degradation of substituted phenols (pentachlorophenol (PCP), trichlorophenol (TCP), dichlorophenol (DCP), and monochlorophenol (CP)). The LangmuirHinshelwood approach was applied to determine oxidation kinetics. Aquasim 2.0 computational software was used to model, simulate and estimate model parameters of the different chlorophenols. Chemical adsorption equilibrium isotherms for the four chlorophenols and phenol were studied and modelled for adsorption onto titanium dioxide $\left(\mathrm{TiO}_{2}\right)$ semiconductor catalyst. Langmuir adsorption parameters were determined and used to calculate adsorption constant and maximum adsorption capacity. The adsorption of chloride phenolics onto titanium dioxide catalyst increased in the order of 4-CP $<$ DCP $<$ Ph $<$ TCP $<$ PCP. Photocatalytic studies analysed the efficiency of oxidation and found improved degradation with higher chloride substituted phenolics in the order of PCP > TCP > DCP $\geq 4$-CP. Photocatalytic parameters were calculated and estimated along with sensitivity and uncertainty analyses.
\end{abstract}

Keywords:

Photocatalysis; chlorophenols; modelling; simulation

\section{Introduction}

Photocatalytic treatment of environmental pollutants such as polychlorinated phenolic chemicals is documented to be effective to the point of complete mineralisation [1-5]. Photocatalysis is an advanced oxidation process (AOP) technology that degrades organic classes of compound at near ambient conditions [5-8]. The simultaneous photocatalytic oxidation of multi-chlorinated phenols has not been researched comprehensively, and the few studies that have been conducted suggest that the chemical oxidation processes involve various complex steps. Different advanced oxidation process techniques utilise different method-specific mechanisms to achieve the degradation process [9-10]. Oxidation dehalogenation of chloride-substituted phenols has been the focus of numerous studies, included are those by Higashimoto et al. [11], Minero et al. [12] and Elsellami et al. [13], these investigated the oxidation mechanisms of 4-chlorophenol and 2.4-dichlorophenol. 
The Langmuir-Hinshelwood (LH) kinetic model has found widespread applications in investigations of advanced oxidation such as photocatalytic oxidation of organic substances. The LH expression can be modified to accommodate many catalytic reactions systems as it satisfies a number of assumptions elaborated in studies and reviews by Fox and Dulay [14], Hoffmann et al. [15], PeraTitus et al. [16], Malato et al. [3], and Augugliaro et al. [17]. The most crucial of these assumptions with regards to application to heterogeneous semiconductor catalyst reactions are those related to surface chemistry. The LH mechanism is founded on the production of electrons $(e-)$ and their pairing holes $\left(h^{+}\right)$from the photo-energy ejection of electrons from the surface of a catalyst. The resulting positive $h^{+}$is trapped by the adsorbed molecules to form a reactive radical state. Photocatalytic reactions are depended on semiconductor surface properties for reduction and oxidation pathways generation in the degradation processes of organic compounds. In this study, the LH kinetic principles will be investigated for their application to the photocatalytic degradation of multisubstituted chloride-halogenated phenols.

Catalyst chemical adsorption capacity is an important step in the degradation process. The adsorption process is a well-established method for the oxidation of organic compounds at low mass concentrations from water, wastewater, and aqueous solutions. Adsorption isotherm parameters of phenol and chloride substituted phenolic compounds will be investigated and modelled using titanium dioxide $\left(\mathrm{TiO}_{2}\right)$ as the semiconductor catalyst of interest. A study by Hamdaoui and Naffrechoux [18] investigated the thermodynamic parameters of adsorption of phenol, 2-CP, 4-CP, 2,4-DCP, and 2,4,6-TCP using various models including Langmuir, Freundlich, Elovich, Temkin, Fowler-Guggenheim, Kiselev, and Hill-de Boer. [18] however used granular activated carbon (GAC) as the adsorbent where-as the current study uses titanium dioxide as an adsorbent and only investigates the Langmuir model, discussion will still draw comparison to [18] in adsorption isothermal parameter determinations. The Langmuir-Hinshelwood mechanism steps and processes are given in Eqs 1-5.

Catalyst + Medium-Pollutant $\longrightarrow$ Catalyst-Pollutant $\underset{\text { desorption }}{\rightleftharpoons}$ Medium-Pollutant

Langmuir equilibrium

Catalyst $+h v \longrightarrow e^{-}+h^{+}$

Pollutant $_{a d s}+h^{+} \longrightarrow$ Pollutant $_{a d s}^{+}$

Pollutant $_{a d s}+e^{-} \longrightarrow$ Pollutant $_{a d s}$

Pollutant $_{\text {ads }}+\longrightarrow$ Medium-Product + Catalyst

Eq.1 shows the adsorption process of the medium bound organic pollutants to the catalyst molecules. The Langmuir equilibrium indicates that there is adsorption-desorption taking place throughout the contact period, and this eventually reaches equilibrium. Eq. 2 is the photo-generation of the electron-hole pair induced by irradiation. Eq. 3 depicts the positive hole trapping process by 
the pollutant molecule. Eq.4 is the decay mechanism of the reactive state, the generated electrons also plays a role in reductive pathway processes not under investigation. Eq. 5 shows the completed oxidation process resulting in a charge-free catalyst and derivative chemical compounds. The photocatalytic degradation sequence of different chlorohalogenated phenolic compounds will be investigated for the influence of substituted chloride ions on the oxidation efficiency. Review articles by Pera-Titus et al. [16], Sharma et al. [5] and others have focused on the treatment of chlorophenols by advanced oxidation processes (AOP) and reported various findings on the influence on the numbers of substituted chloride halogens to the oxidation sequence. Simulated kinetic models will be performed and modelled to estimated parameters by adapting the LH expression.

\section{Experimental}

\subsection{Materials}

4-chlorophenol (4-CP) 99\% purity, 2.4-dichlorophenol (2.4-DCP) 99\%, 2.4.6-trichlorophenol (2.4.6TCP) 98\%, pentachlorophenol (PCP) 97\%, and anatase 99.7\% titanium dioxide $\left(\mathrm{TiO}_{2}\right)$ were purchased from Sigma-Aldrich Logistik GmbH (Schnelldorf, Germany). Phenol, GC grade methanol, sulphuric acid (95-99\%) and sodium hydroxide were purchased from Merk (South Africa). Ultra-pure (UP) water was dispensed by the Millipore Direct Q3 with pump instrument. Reference standard were purchased from PerkinElmer (South Africa division).

\subsection{Equilibrium isotherms}

Adsorption isotherms were determined by; accurately weighed anatase titanium dioxide $\left(0.03 \mathrm{~g} \mathrm{~L}^{-1}\right)$ dispensed in aqueous chlorophenols spiked $\left(5,10,1520,25 \mathrm{mg} \mathrm{L}^{-1}\right)$ solutions $(0.5 \mathrm{~L})$ continuously stirred at $300 \mathrm{rpm}$. Pentachlorophenol has low solubility in aqueous solutions ( $20 \mathrm{mg} \mathrm{L}^{-1}$ at $30{ }^{\circ} \mathrm{C}$ ), therefore lower chemical concentrations within the PCP solubility range were used, as given above. The temperature was controlled at $19{ }^{\circ} \mathrm{C}$. The resulting $\mathrm{pH}$ of the solutions was in within the range of 5.4 to 5.7. The solutions were agitated for over 20 hours to reach equilibrium. After a minimum period of 20 hours samples were removed and centrifuged (eppendorf AG minispin) for 30 minutes at $5000 \mathrm{rpm}$. Sample residual concentrations were analysed using the GCMS. Experiments were repeated and average values are reported. Langmuir isotherm models were used to calculate adsorption parameters.

\subsection{Photocatalytic reactors installation and setup}

The setup was installed in a temperature regulated walk-in reactor room that is wall-connected to a cold-room of $4{ }^{\circ} \mathrm{C}$ set temperature, the wall connection has small port-openings that allow transfer of cooled air. The reactor room has a driven air-vacuum that constantly drives the cooling system. The reactor system make-up rested on a bench structure. Four $400 \mathrm{~mL}$ transparent glass reactors vessels were mounted on stands side-by-side (30 cm apart). Each vessel had inlets mounted on the underside for oxygen $\left(\mathrm{O}_{2}\right)$ of $99 \%$ purity introduction connected to a regulator. In between the four 
vessels were 3 quartz glass sleeves mounted equidistant from each vessel. Encased in the quartz sleeves were long-arc 400 Watts (Philips HOK 4/120 SE) medium pressure lamps. The two reactor vessels on the ends (labelled $A$ and $D$ ) received radiation source from single lamps, while the two reactors in between were exposed to radiation source from both directions (labelled $B$ and $C$ ). Maximum irradiation exposure of 630 minutes (10.5 hours), sampling performed at predetermined time intervals.

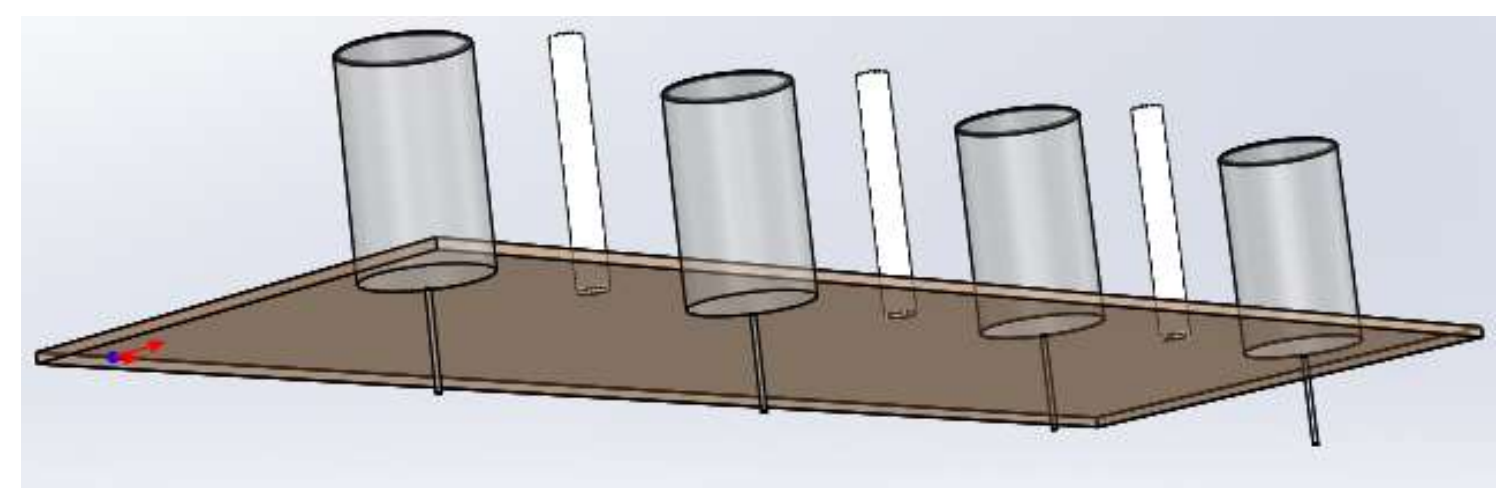

Fig.1. Three-dimensional reactor configuration schematic representation. Radiation lamps power ballasts, connecting tubing and wiring, air flow-meters, and vessel contents not included.

\subsection{Analysis}

The polychlorinated phenolics and derivative products were analysed using a gas chromatography (GC) system comprising of a clarus $600 \mathrm{GC}$, clarus $600 \mathrm{~T}$ mass spectrometer (MS), attached to a turbomatrix 40 trap headspace sampler (PerkinElmer, South Africa division). The chemical separation component was the Elite $5 \mathrm{MS}$ GC system capillary column $(30 \mathrm{~m}, 250 \mu \mathrm{m})$ from PerkinElmer. Helium (He) carrier gas of 99.999\% purity and applied at a flow rate of $1 \mathrm{~mL} / \mathrm{min}$. MS interface comprised of an Electron loniser (EI) and a high performance mass analyser.

\subsection{Data process and modelling}

Sigmaplot 11 scientific data analysis graphics computation software was used for data processing and statistical analysis. Aquasim 2.0 computer program for the identification and simulation of aquatic systems was used to simulate and model the photocatalytic oxidation profiles, and parameter estimations. 


\section{Results}

\subsection{Adsorption Isotherms}

The experimental kinetic adsorption isotherms of chlorophenols and phenol from aqueous solutions onto titanium dioxide are presented in Fig.2. Table 1 presents the calculated parameters for the adsorption of the phenolic compounds onto titanium dioxide surface sites. With the exception of phenol, the four tested chlorophenols appear to be adsorbed on to the catalyst in concentrations directly proportional to their degree of chlorination. [18] reported similar graphical findings of chemical isothermal profiles. According to Giles et al. [19], the nature of curvature shape observed in the experiments indicates that as more sites in the substrate are filled it becomes increasingly difficult for a bombarding solute molecule to find vacant sites. Though the range of adsorption profiles in the current study is smaller than that investigated by [18], the findings show a similar pattern for 4-chlorophenol, dichlorophenol and trichlorophenol. Excluding phenol, the order of adsorption onto titanium dioxide is 4-CP $<$ DCP $<\mathrm{TCP}<\mathrm{PCP}$. The plots of the inverse of the adsorbed chemical concentration per weight $\left(Q_{e}\right)$ versus the inverse of the final equilibrium solution concentration $\left(C_{e}\right)$ were used for studying the adsorption data. The Langmuir adsorption constants $\left(K_{L}\right)$ for all compounds were calculated as recorded in Table 1.

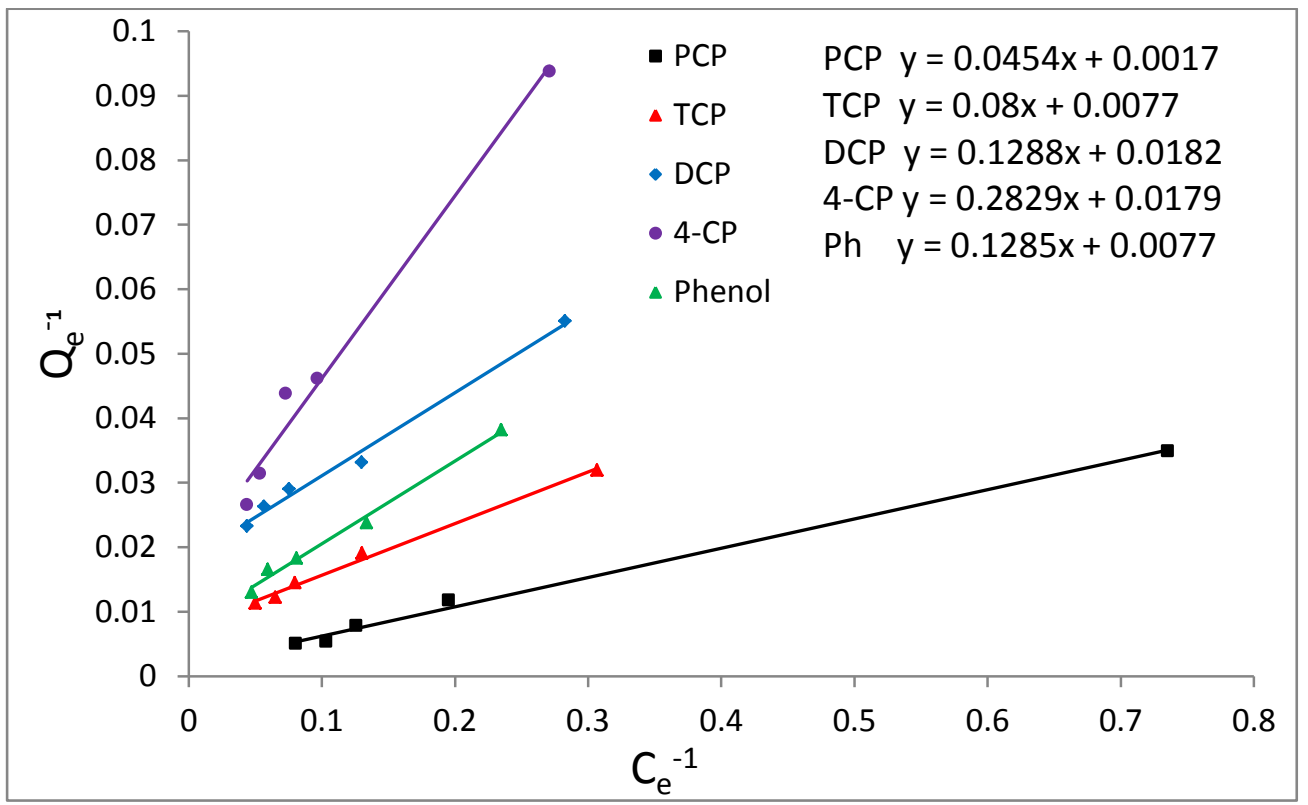

Fig.2. Langmuir isotherm plots for pentachlorophenol, trichlorophenol, dichlorophenol, chlorophenol and phenol.

Table 1. Parameters of Langmuir isotherms for the adsorption of phenolic compounds onto $\mathrm{TiO}_{2}$

\begin{tabular}{lccccc}
\hline Isotherm & Phenol & 4-CP & DCP & TCP & PCP \\
\hline & & & & & \\
$\boldsymbol{K}_{\mathbf{L}}\left(\mathrm{mg} \mathrm{L}^{1}{ }^{1}\right)$ & 0.0603 & 0.0676 & 0.1420 & 0.0987 & 0.0390 \\
$\boldsymbol{Q}_{m}\left(\mathrm{mg} \mathrm{g}^{-}\right)$ & 130.01 & 57.470 & 55.860 & 130.02 & 588.20 \\
$\boldsymbol{R}^{2}$ & 0.9919 & 0.9839 & 0.9913 & 0.9938 & 0.9956 \\
Stdev & 0.0060 & 0.0149 & 0.0155 & 0.00984 & 0.00595 \\
\hline
\end{tabular}


3.2. Experimental models of simultaneous photocatalytic degradation of multi-chloride substituted phenolics in batch systems.

Few investigations have been conducted on the behaviour of chloride-halogen substituted phenol compounds simultaneously degraded photocatalytically, and even less available information on the modelled and simulated oxidation of the photocatalytic process. Investigations on the advanced oxidation process of simultaneously degraded chlorinated phenolic compounds present an unclear picture of the degradation sequence of the compounds. Song-hu and Xiao-hua [20] obtained a Fenton's reagent sequence of 2.4-DCP > 2.4.6-TCP > PCP > 4-CP, Bandara et al. [21] obtained a 2.4$\mathrm{DCP}>2$ 2-CP $>2.3-\mathrm{DCP}>2.4 .6-\mathrm{TCP}$ photocatalytic degradation sequence, and Benitez et al. [22] recorded a sequence of 2.3.4.6-TeCP $>$ 2.4.6-TCP $>$ 2.4-DCP $>4$-CP under ozonation. Four chlorophenols were simultaneously oxidised in open batch systems in this study. The hypothesised mechanism is that the degradation processes of individual compounds are coupled with the conversion and formation of derivative species. A higher level substituted chlorophenol is expected to undergo oxidation resulting in the decrease in its concentration and a subsequent formation of a lower level substituted compound in combination with other unrelated derivative species. This is in contrast to findings in studies where the photocatalytic process resulted in higher chloride halogenated phenols recording slower rates of degradation and contrary to expectation where simpler organic compound complexes are typically broken down faster and easier than the higher complexes of organic groups. The implication of the current hypothesis is that the resulting concentration values of individual compounds at different time intervals would encompass; (a) the degradation of each compound that is dependent on the degradation kinetics as though the compound were by itself in a batch, (b) the formation of that particular compound as a derivative from the conversion of the higher substituted phenolic, with the exception of the highest substituted compound, (c) and the accumulative effect of the derived lower levels substituted compounds coupled with their initial concentration and individual kinetic dependent degradation. There are also intermediate chlorophenol compounds which do not form part of the compounds of interest in this study, such as 2-chlorophenol, 2.3-dichlorophenol and 2.3.4.6-tetrachlorophenol that may influence the degradation and conversion processes. Analyses of experimental results are performed using kinetic simulations of the Langmuir-Hinshelwood model. Kinetic expression parameters are estimated and evaluated.

The proposed mechanism given by Augugliaro et al. [17] for the photocatalytic degradation of aromatic compounds such as polychlorophenols is the substitution of chloro-halide species by hydroxyl groups. This generates a single less halogenated chlorophenol compound. Eq.6 shows the intermediate substitution step of the dechlorination of pentachlorophenol to tetrachlorophenol. The oxidation of the hydroxyl-group results in the introduction of tetrachlorophenol to the system. The overall stoichiometric mineralisation process of pentachlorophenol is provided in Eq.7. Eq.8. is a compound-specified Langmuir-Hinshelwood expression, each variable has a compound subscript letter to differentiate different chlorophenols, and the subscripts in Eq.8 specify pentachlorophenol. 
<smiles>Oc1c(Cl)c(Cl)c(Cl)c(Cl)c1Cl</smiles>

PCP

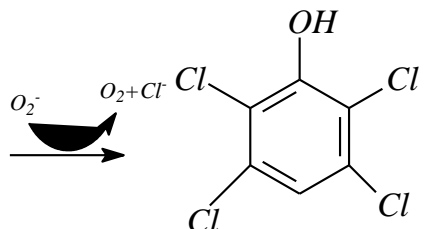

2.3.5.6-TeCP

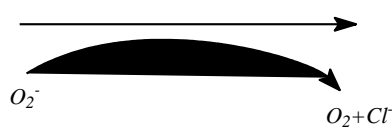

$\mathrm{O}_{2}+\mathrm{Cl}$
(6)

Figs.3A to 3D are graphical plots of the photocatalytic degradation profiles of the polychlorinated phenols. The estimated parameters of the models are recorded in Table 2. The general qualitative outlook of the 4 reactors suggests that reactors $A$ and $D$ performed less efficiently than reactors $B$ and $C$, this is expected due to the amount of ultra-violet radiation exposed to the inner-lying reactors in comparison to the outer-lying reactors. Eq. 8 is the degradation expression used for the derivation of pentachlorophenol photocatalytic kinetics. In all the reactor systems, though being the largest and most complex polychlorinated phenol chemical, pentachlorophenol recorded the highest photocatalytic efficiency. Pentachlorophenol's subsequent degradation as shown in Eq.6 results in the conversion and formation of a lower level chlorinated compound (TeCP) amongst other derivative chemicals not included in the equation. The formation and concentration contributions from higher level chlorinated compounds to lower level chlorinated compounds are theoretically challenging to quantify. In this study, experimental data based models were used to estimate the contribution of higher level chlorinated phenolics to lower level compounds by applying chemical simulation techniques. An accumulation expression is adapted into the $\mathrm{LH}$ model as a function of concentration and the rate of formation of lower derived chlorophenols at the expense of source compounds. 


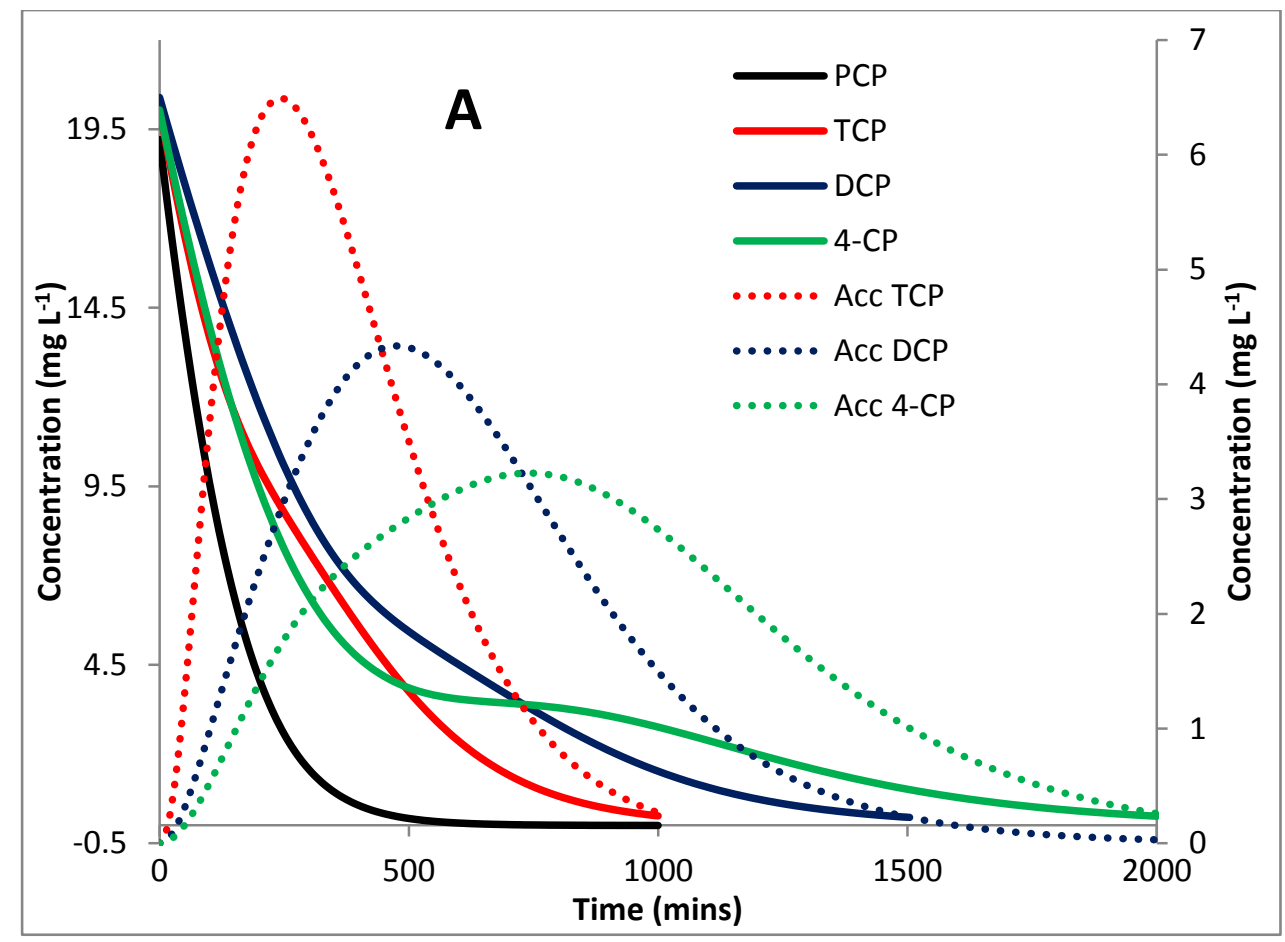

Fig.3A. Simulated simultaneous photocatalytic degradation profiles of multi-chlorinated substituted phenolics in reactor A. PCP (primary axis), TCP (primary axis), DCP (primary axis), 4-CP (primary axis), accumulated TCP (secondary axis), accumulated DCP (secondary axis), accumulated 4-CP (secondary axis).

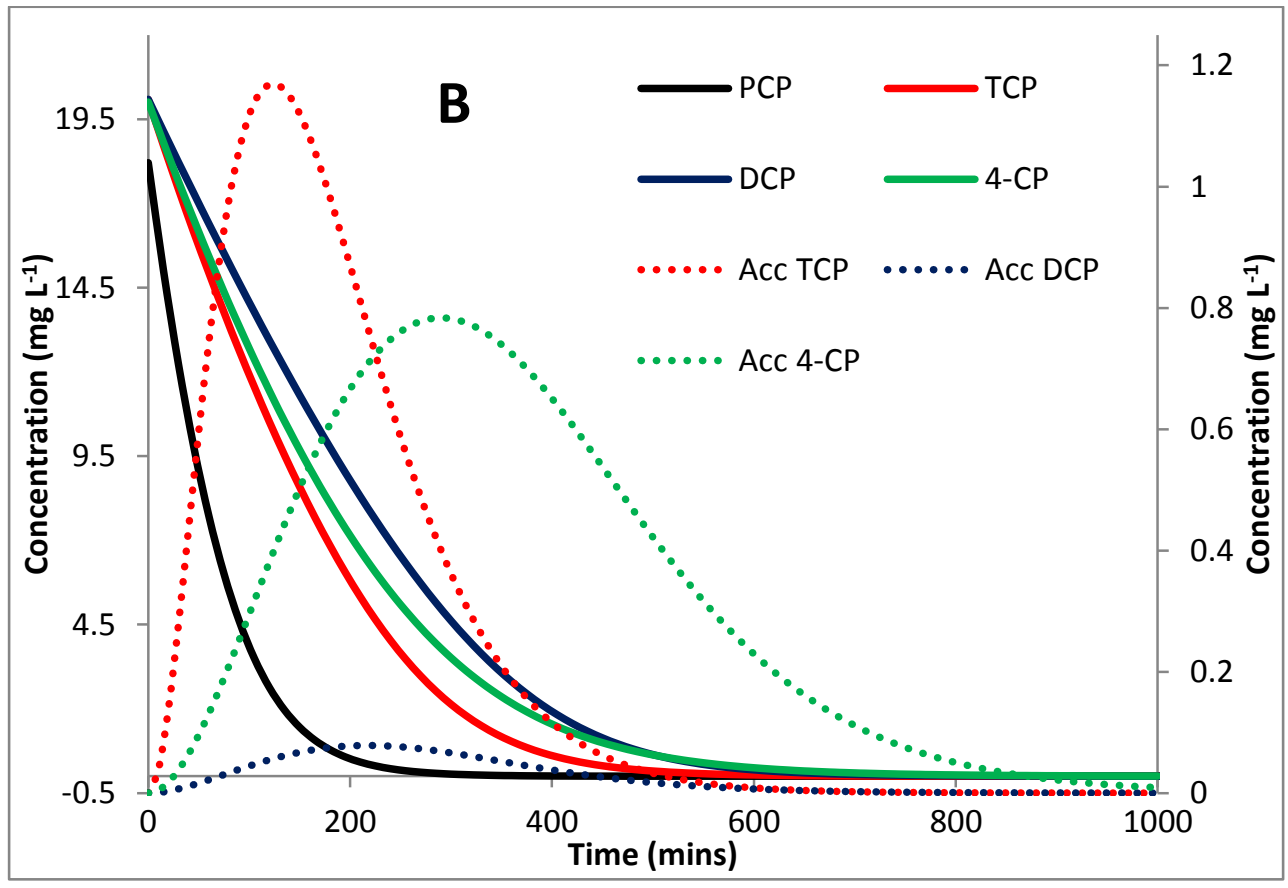

Fig.3B. Simulated simultaneous photocatalytic degradation profiles of multi-chlorinated substituted phenolics in reactor B. PCP (primary axis), TCP (primary axis), DCP (primary axis), 4-CP (primary axis), accumulated TCP (secondary axis), accumulated DCP (secondary axis), accumulated 4-CP (secondary axis) 


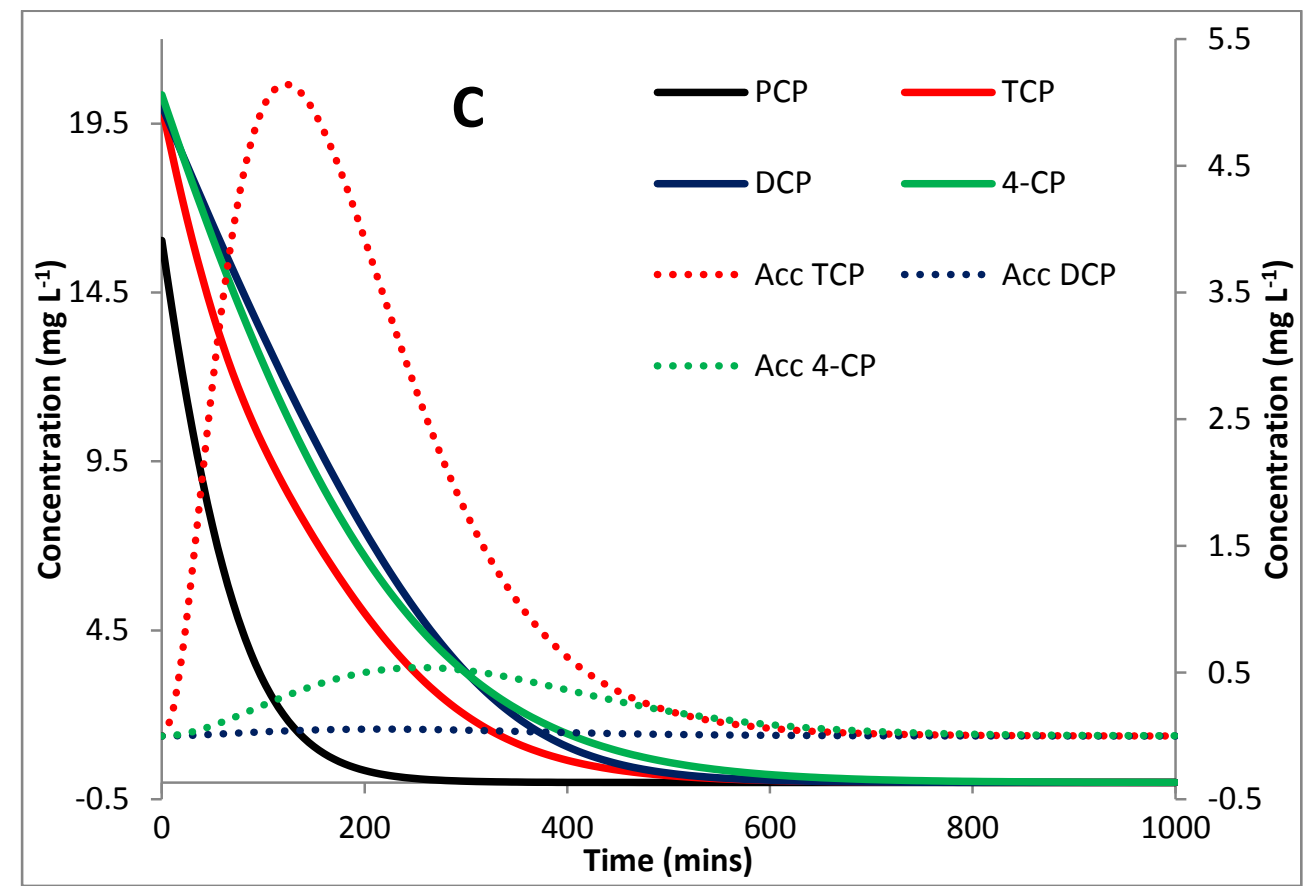

Fig.3C. Simulated simultaneous photocatalytic degradation profiles of multi-chlorinated substituted phenolics in reactor C. PCP (primary axis), TCP (primary axis), DCP (primary axis), 4-CP (primary axis), accumulated TCP (primary axis), accumulated DCP (secondary axis), accumulated 4-CP (secondary axis)

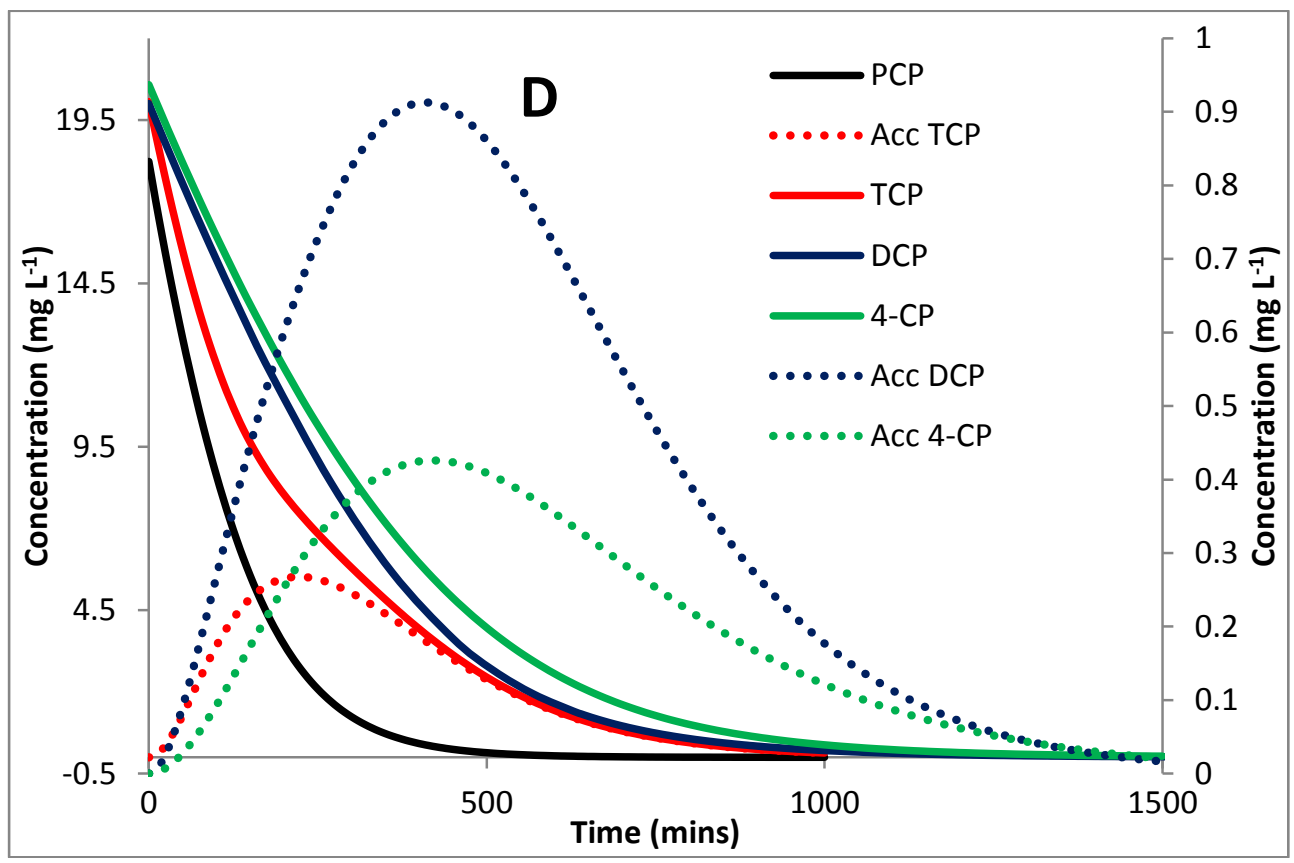

Fig.3D. Simulated simultaneous photocatalytic degradation profiles of multi-chlorinated substituted phenolics in reactor D. PCP (primary axis), TCP (primary axis), DCP (primary axis), 4-CP (primary axis), accumulated TCP (primary axis), accumulated DCP (secondary axis), accumulated 4-CP (secondary axis 
Table 2. Photocatalytic estimated parameters of phenolic compounds using the LangmuirHinshelwood

\begin{tabular}{|c|c|c|c|c|}
\hline Parameter & 4-CP & DCP & TCP & PCP \\
\hline \multicolumn{5}{|l|}{ Reactor A } \\
\hline$K_{L}\left(\mathrm{mg} \mathrm{L} \mathbf{L}^{1}\right)$ & 0.06756 & 0.14646 & 0.10892 & 0.03890 \\
\hline$k\left(\mathrm{mg} \mathrm{L}-{ }^{1} \mathrm{~min}-{ }^{1}\right)$ & 0.11668 & 0.06560 & 0.11108 & 0.27392 \\
\hline$k_{2}\left(\mathrm{mg} \mathrm{L}^{1}{ }^{1} \min ^{1}{ }^{1}\right)$ & 0.00092 & 0.00150 & 0.00500 & \\
\hline$C_{o}\left(\mathrm{mg} \mathrm{L}^{1}\right)$ & 20.20432 & 20.3906 & 20.7000 & 19.2000 \\
\hline \multicolumn{5}{|l|}{ Reactor B } \\
\hline$K_{L}\left(\mathrm{mg} \mathrm{L}^{1}{ }^{1}\right)$ & 0.06961 & 0.15766 & 0.10912 & 0.04055 \\
\hline$k\left(\mathrm{mg} \mathrm{L}^{-1} \mathrm{~min}^{-1}\right)$ & 0.13832 & 0.08297 & 0.13423 & 0.52727 \\
\hline$k_{2}\left(\mathrm{mg} \mathrm{L}^{-1} \mathrm{~min}^{1}{ }^{1}\right)$ & 0.00051 & $7.19 E-5$ & 0.00315 & \\
\hline$C_{o}\left(\mathrm{mg} \mathrm{L}-^{1}\right)$ & 20.0052 & 20.0327 & 20.0104 & 18.2108 \\
\hline \multicolumn{5}{|l|}{ Reactor C } \\
\hline$K_{L}\left(\mathrm{mg} \mathrm{L}^{1}{ }^{1}\right)$ & 0.06961 & 0.15766 & 0.09300 & 0.04505 \\
\hline$k\left(\mathrm{mg} \mathrm{L-}{ }^{1} \mathrm{~min}-{ }^{1}\right)$ & 0.15237 & 0.09444 & 0.22212 & 0.50108 \\
\hline$k_{2}\left(\mathrm{mg} \mathrm{L}-{ }^{1} \min -{ }^{1}\right)$ & 0.00041 & $6.69 \mathrm{E}-5$ & 0.01066 & \\
\hline$C_{o}\left(\mathrm{mg} \mathrm{L}^{1}\right)$ & 20.3503 & 20.0327 & 20.0104 & 16.0400 \\
\hline \multicolumn{5}{|l|}{ Reactor D } \\
\hline$K_{L}\left(\mathrm{mg} \mathrm{L}^{-1}\right)$ & 0.06897 & 0.13277 & 0.09486 & 0.03748 \\
\hline$k\left(\mathrm{mg} \mathrm{L}-{ }^{1} \mathrm{~min}-{ }^{1}\right)$ & 0.08222 & 0.06886 & 0.15253 & 0.29565 \\
\hline$k_{2}\left(\mathrm{mg} \mathrm{L}^{1} \mathrm{~min}^{1}{ }^{1}\right)$ & 0.00027 & 0.00053 & 0.00493 & \\
\hline$C_{o}\left(\mathrm{mg} \mathrm{L}^{1}\right)$ & 20.5844 & 20.0014 & 20.0584 & 18.2251 \\
\hline
\end{tabular}

This is used to estimated and predict parameters of lower level compounds of interest. Eq. 9 is the adapted Langmuir-Hinshelwood expression, where $k_{n}$ is the photocatalytic rate constant $\left(\mathrm{mg} \mathrm{L}^{-1} \mathrm{~min}^{-}\right.$ $\left.{ }^{1}\right), n$ is the compound under scrutiny (c 4-CP, $d$ DCP, $t$ TCP, $p$ PCP), $K_{L n}$ is the free energy Langmuir adsorption constant $\left(\mathrm{mg} \mathrm{L}^{-1}\right), C_{n}$ is the concentration of the analyte under scrutiny $\left(\mathrm{mg} \mathrm{L}^{-1}\right), C_{n+1}$ concentration of the higher level chlorophenol present in solution $\left(\mathrm{mg} \mathrm{L}^{-1}\right), k_{n 2}$ is the formation constant of the lower level chlorophenol $\left(\mathrm{mg} \mathrm{L}^{-1} \mathrm{~min}^{-1}\right)$. Langmuir-Hinshelwood expression modifications of Eq. 9 are essentially focused on the total compound concentration $(n)$ present in solution, which is the un-oxidised concentration remaining from the initial prepared solution and the addition of process derived chlorophenols taking into accounts principles of the exponential chemical growth to maximum model. Fig.4 shows the simulated photocatalysis of the different chlorophenols of reactor $A$ on a single $y$-axis. The dotted profiles are the estimated increases in concentrations of lower level chlorophenols at the expense of higher chlorinated phenols. When Fig.4 is viewed closely, the data indicates three situations; (i) degradation of higher chlorinated compounds (PCP) alongside simultaneous degradation of lower level chlorinated compounds (TCP) is observed, (ii) the profile differentiation of lower level substituted chlorophenols from higher levels substituted chlorophenols due to formation and accumulation resulting from the converted higher 


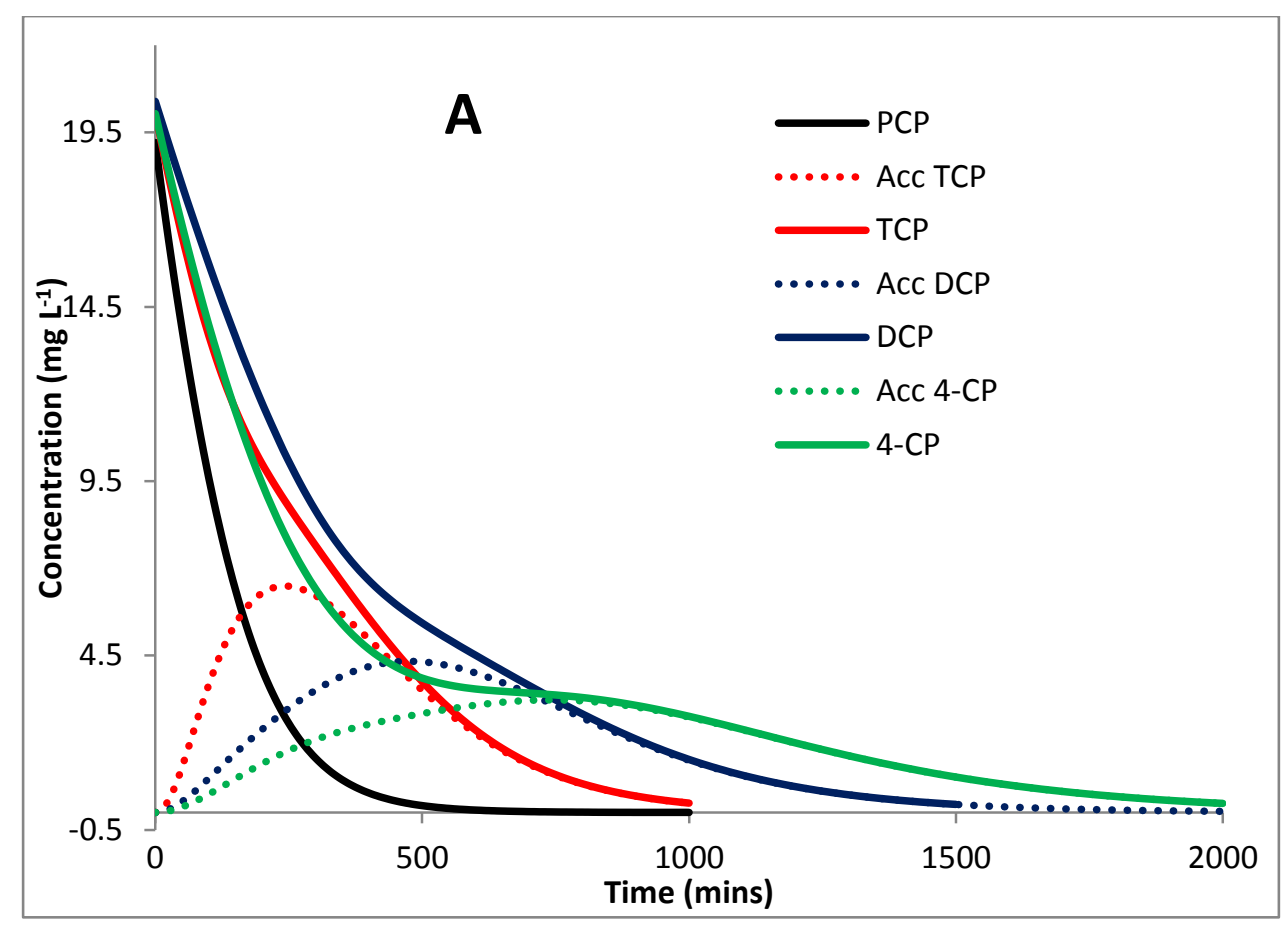

Fig. 4. Simulated simultaneous photocatalytic degradation profiles of multi-chlorinated substituted phenolics in reactor A with the exclusion of secondary axis.

substituted compounds, this is indicated by the bloated middle section of the profile, (iii) upon complete degradation and conversion of the higher level chlorophenol such as PCP, an improved removal efficiency of the lower level chlorinated compound such TCP is seen towards near zero point. This phenomenon is similar for the remaining chlorophenols in solution, the difference lies in the rate of degradation of each chlorophenol and the rate of introduction of the compound from the converted and depleted higher substituted compounds. Figs.3A to 3D represents the photocatalytic profiles with the estimated converted and accumulative concentrations conveyed on the secondary axis. The chlorophenol degradation sequence appears to differ slightly between the reactors $A$ and $D$ (outbound, exposed to a single lamp, one direction), and reactors $B$ and $C$ (inbound, exposed to two lamps, two directions). Reactors $A$ and $D$ have a photocatalytic sequence $\mathrm{PCP}<\mathrm{TCP}<\mathrm{DCP}<4-\mathrm{CP}$ while reactors $B$ and $C$ have a photocatalytic sequence of $P C P<T C P<4-C P \leq D C P$. The amount of available irradiation influences the photocatalytic process, the oxidation efficiency of chlorophenols from reactors irradiated from both sides is better than that of the reactors from one side. The rate of degradation for reactors $B$ and $C$ is much improved compared to reactors $A$ and $D$, the accumulation effect in minimised due to faster removal and impeded conversion. Quantitative data in Table 2 showing estimated kinetic parameters suggests that the introduction of derivative compounds in reactors $B$ and $C$ is offset by the significantly higher rate of degradation. 4-chlorophenol and 2.4dichlophenol present an interesting dynamic, recorded experimental data suggest that 4-CP has a higher propensity for oxidation than DCP under the experimental conditions, though 4-CP is a derivative compound of the conversion process of DCP. Though 4-CP is constantly introduced in solution from the conversion of DCP, it is only in the latter periods of illumination where a distinct separation takes place, reactor $A$ and $D$ better depict this relationship. 
Table 3. Photocatalytic oxidation derived and intermediate organic chemicals

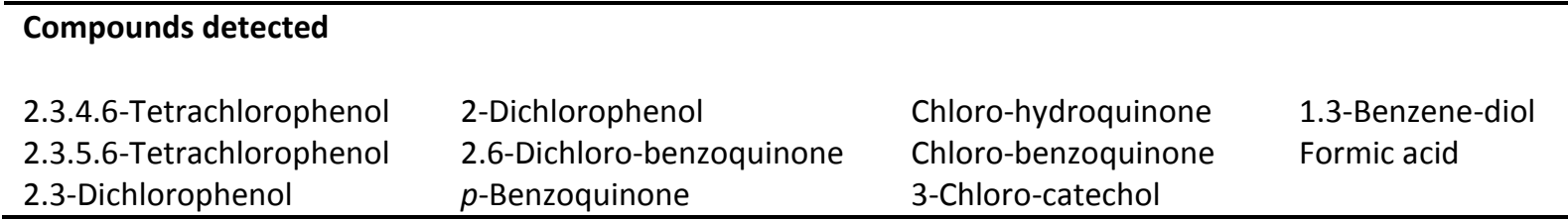

A brief literature search was conducted, and a list of reported oxidation derived chemicals and mechanism detected intermediates compounds were compiled from studies of polychlorosubstituted phenolics. The mass spectrum (MS) skeletons highest ten ions of each of the compounds listed, through the Perkin Elmer Turbomass software and the NIST Library software, were processed against thirty randomly selected relevant experimental data sets using the complete set of chromatograms per sampling interval unit for the duration determined. The processing was aided by the fact that all chlorophenols were simultaneously analysed, meaning that all listed spectrum ions were processed for entire mass scan periods. Barring the spectrum hits on the chlorophenol analytes under project investigation, Table 3 shows the only organic compound detected using the list compiled from the chromatograms scanned. All compounds in Table 3 were present in trace amounts, it is only with the aid of the total ion chromatogram (TIC) analyser that each peak with the relevant ions can be identified.

\subsection{Experimental parameter sensitivity analyses models}

The goal of sensitivity analysis is to check if model parameters can be uniquely determined with the aid of the available data and to estimate the uncertainty of the parameter estimates [23]. This is done by estimating the standard errors and correlation coefficients of parameters during the parameter estimation procedure. Sensitivity function in this application measures the absolute change per unit of each parameter with relative change in time. The partial derivatives required for the calculation of sensitivity functions are calculated by comparing the results obtained with the original parameter value and those obtained with a parameter value that deviates from the original value by a given percentage of its standard deviation. Standard deviation values of 10 percent were used for parameters where the actual values were not possible to calculate from experimental results. Figs.5.1.1 to 5.4.2 show time dependence of the sensitivity functions of calculated concentrations with respect to model parameters of initial concentration $\left(C_{n o}\right)$, Langmuir adsorption constant $\left(K_{L n}\right)$, photocatalytic constant $\left(k_{n}\right)$, and formation constants $\left(k_{n 2}\right)$ where applicable for each chlorophenol. Fig.5.1.1 shows the sensitivity analyses in reactor $A$, the parameter $C_{n o}$ of the different chlorophenols is related to the measured concentrations. There are fractional increases in initial concentration with discreet changes in time for TCP, DCP and 4-CP. It is interesting to note that the $C_{c o}$ measures smaller relative changes in time in comparison to $C_{d o}$, this can be explained by the significantly higher estimated degradation constant of $0.11668\left(k_{c}\right)$ than $0.06560\left(k_{d}\right)$. The sensitivity functions of the parameters $K_{L n}$ and $k_{n}$ have a similar shape. The negative signs indicate that the 


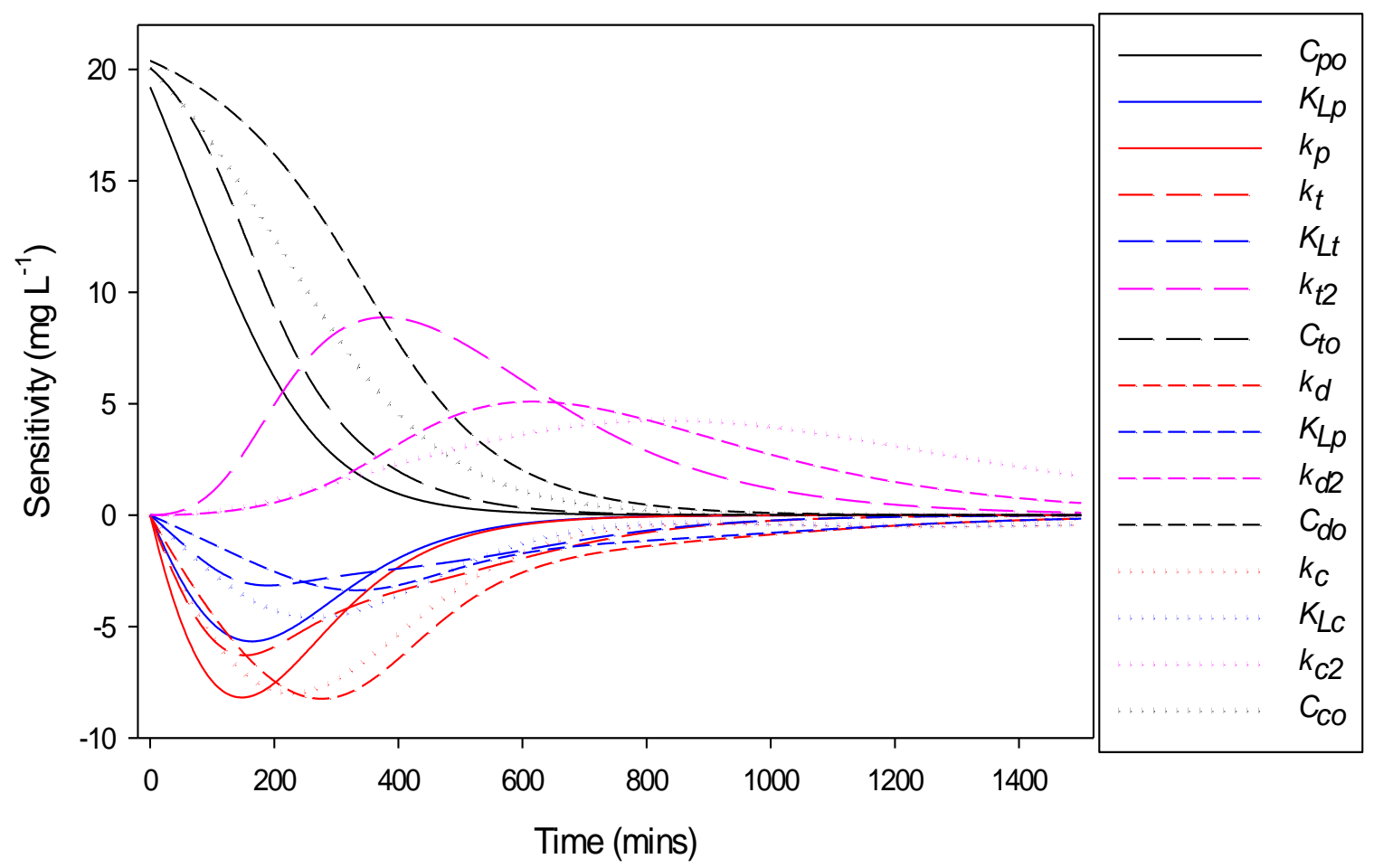

Fig.5.1.1. Time course of the sensitivity functions of $C_{p}, C_{t}, C_{d}$ and $C_{c}$ with respect to LangmuirHinshelwood kinetics in reactor A.

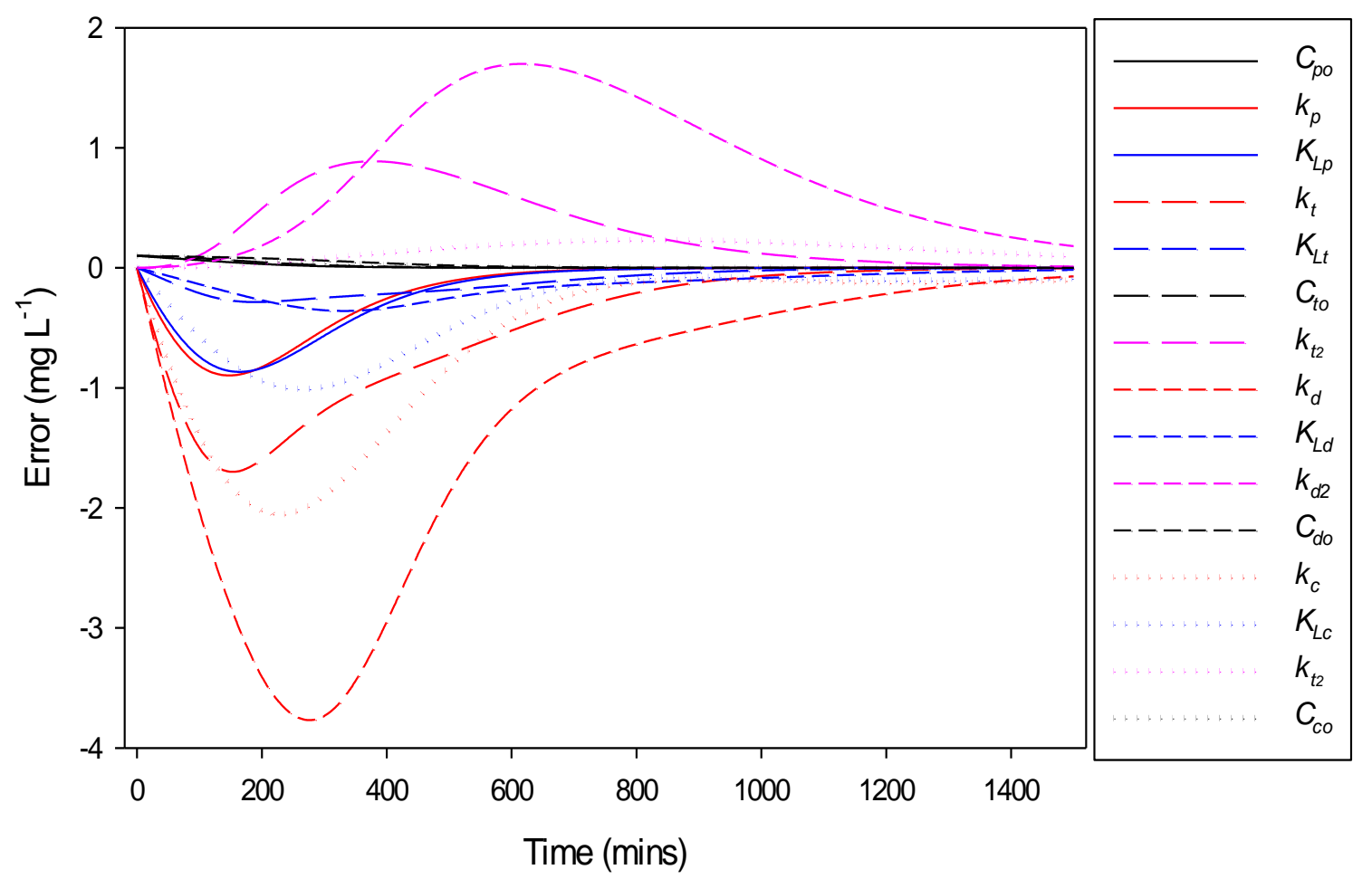

Fig.5.1.2. Error contributions of model parameters on the calculated concentrations of $C_{p}, C_{t} C_{d}$ and $C_{c}$ with respect to Langmuir-Hinshelwood kinetics in reactor $A$. 


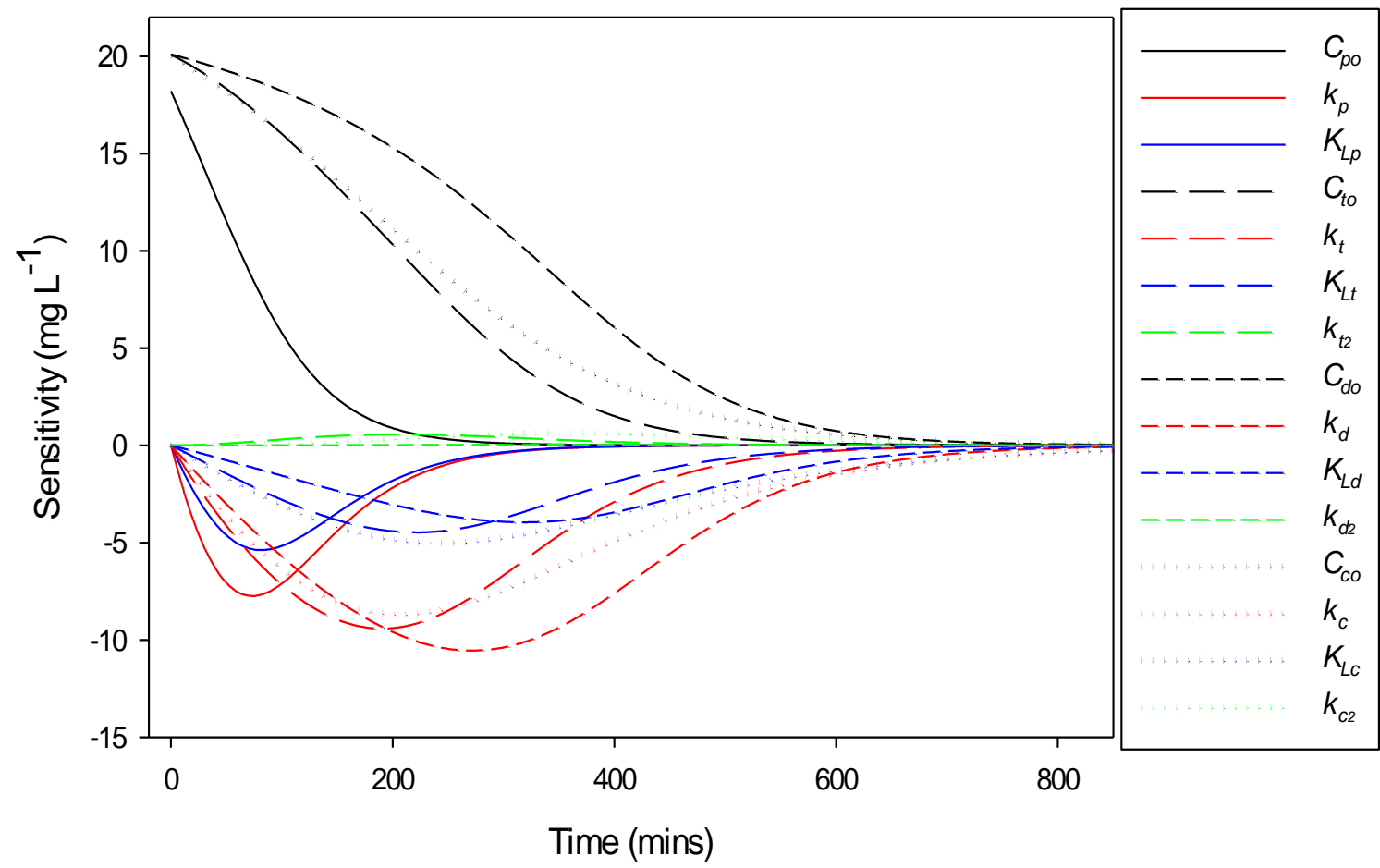

Fig.5.2.1. Time course of the sensitivity functions of $C_{p}, C_{t}, C_{d}$ and $C_{c}$ with respect to LangmuirHinshelwood kinetics in reactor $B$.

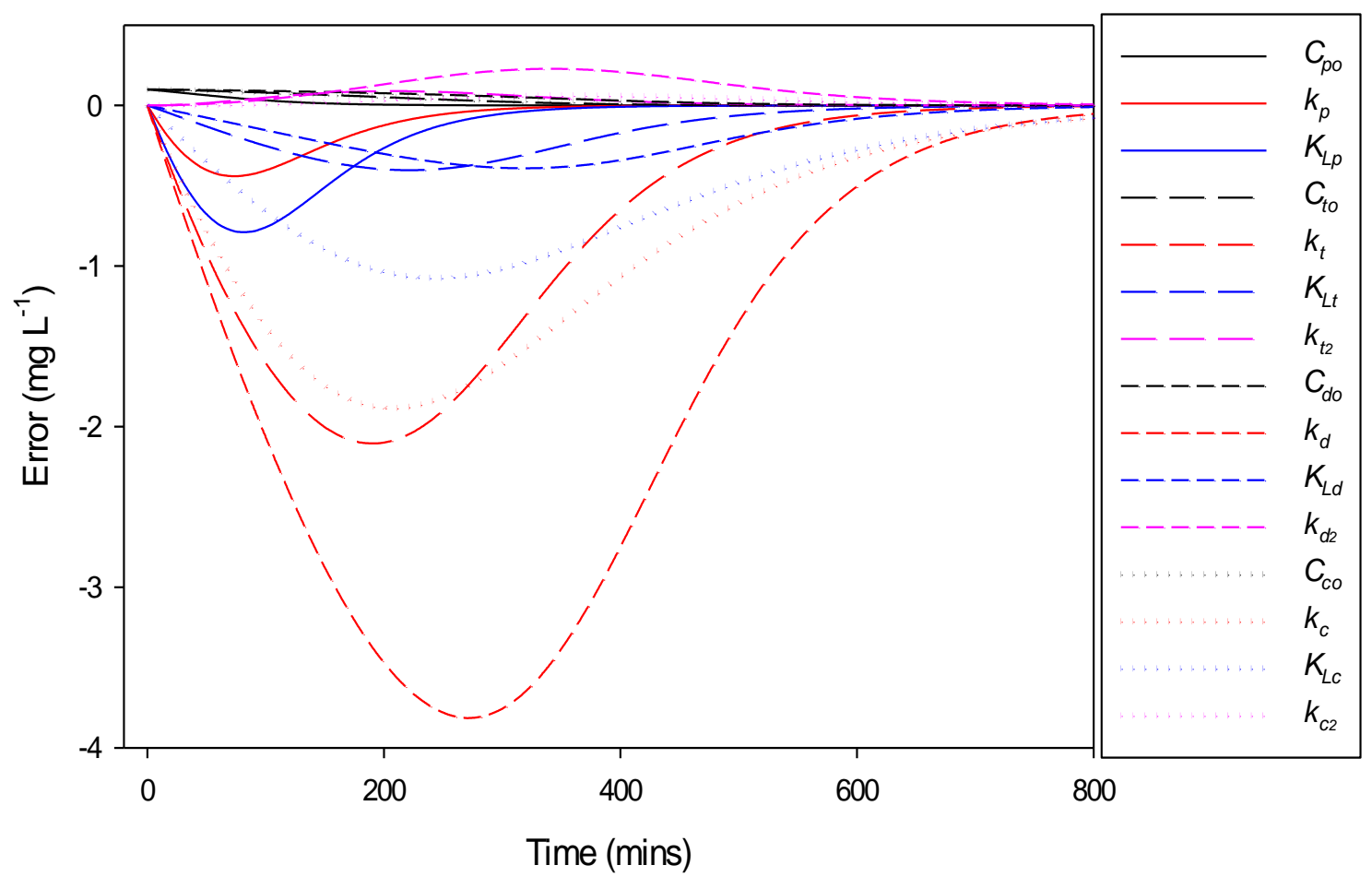

Fig.5.2.2. Error contributions of model parameters on the calculated concentrations of $C_{p}, C_{t} C_{d}$, and $C_{c}$ with respect to Langmuir-Hinshelwood kinetics in reactor $B$. 


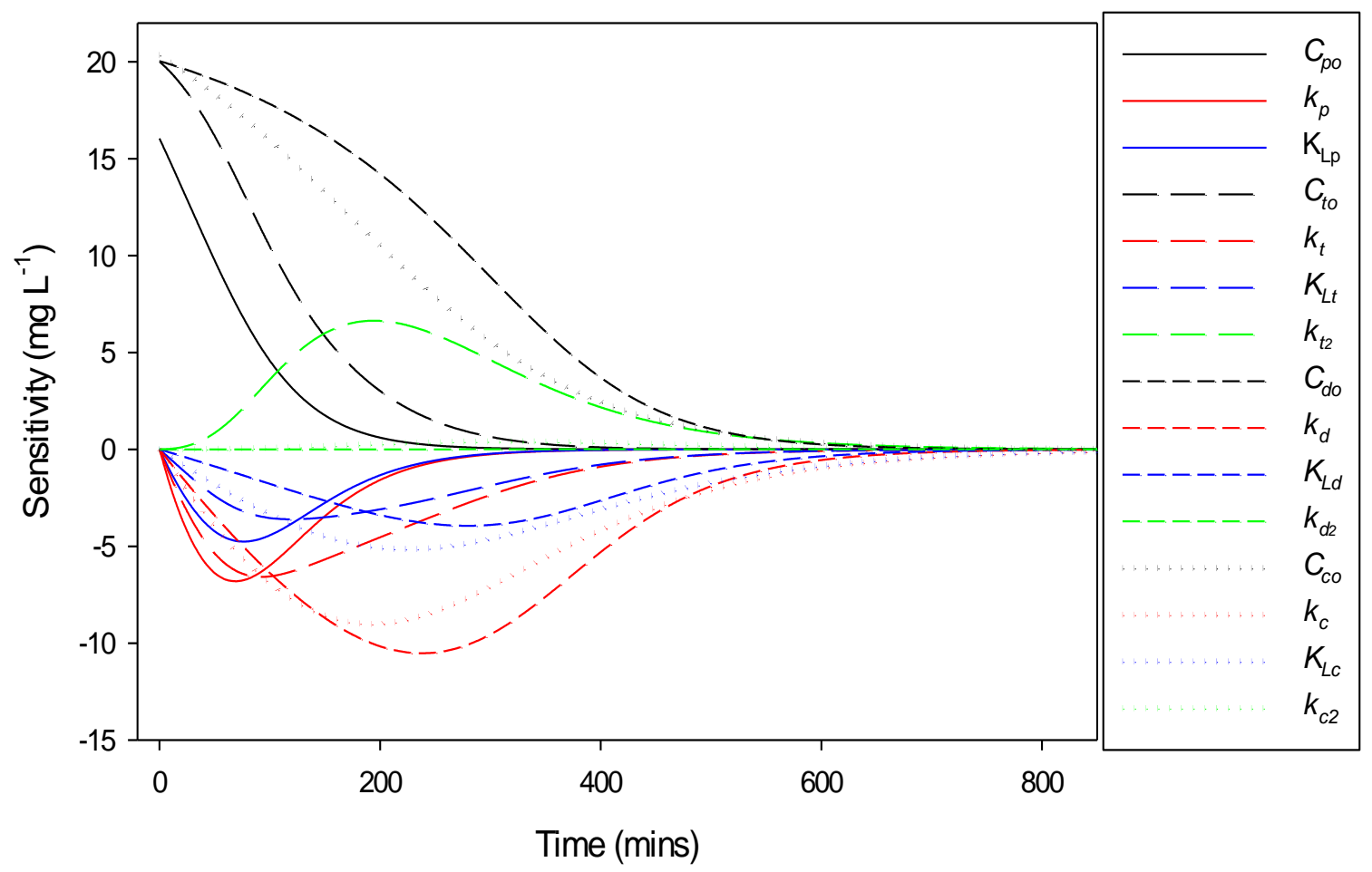

Fig.5.3.1. Time course of the sensitivity functions of $C_{p}, C_{t}, C_{d}$ and $C_{c}$ with respect to LangmuirHinshelwood kinetics in reactor $\mathrm{C}$.

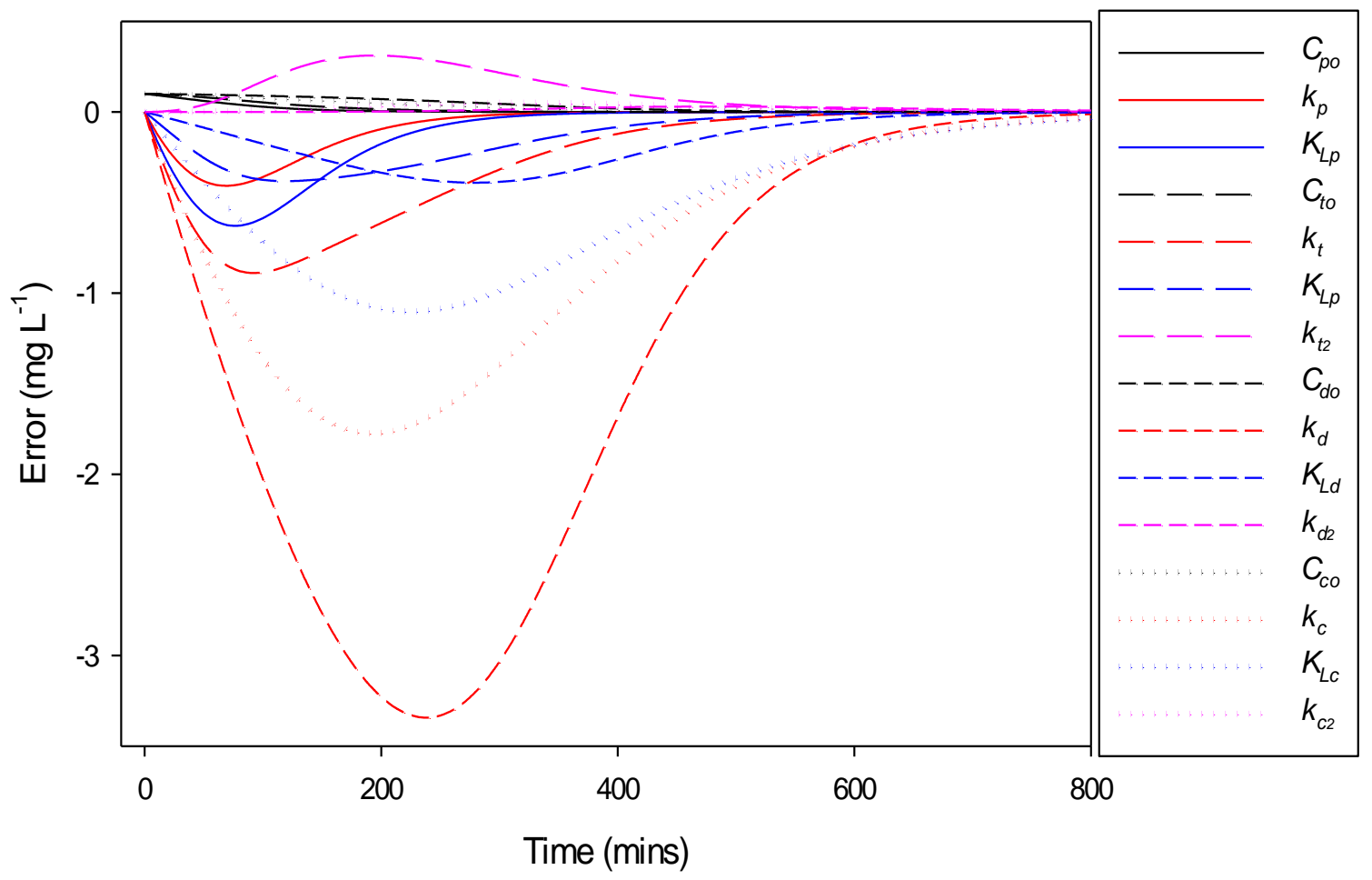

Fig.5.3.2. Error contributions of model parameters on the calculated concentrations of $C_{p}, C_{t} C_{d}$ and $C_{c}$ with respect to Langmuir-Hinshelwood kinetics in reactor $\mathrm{C}$. 


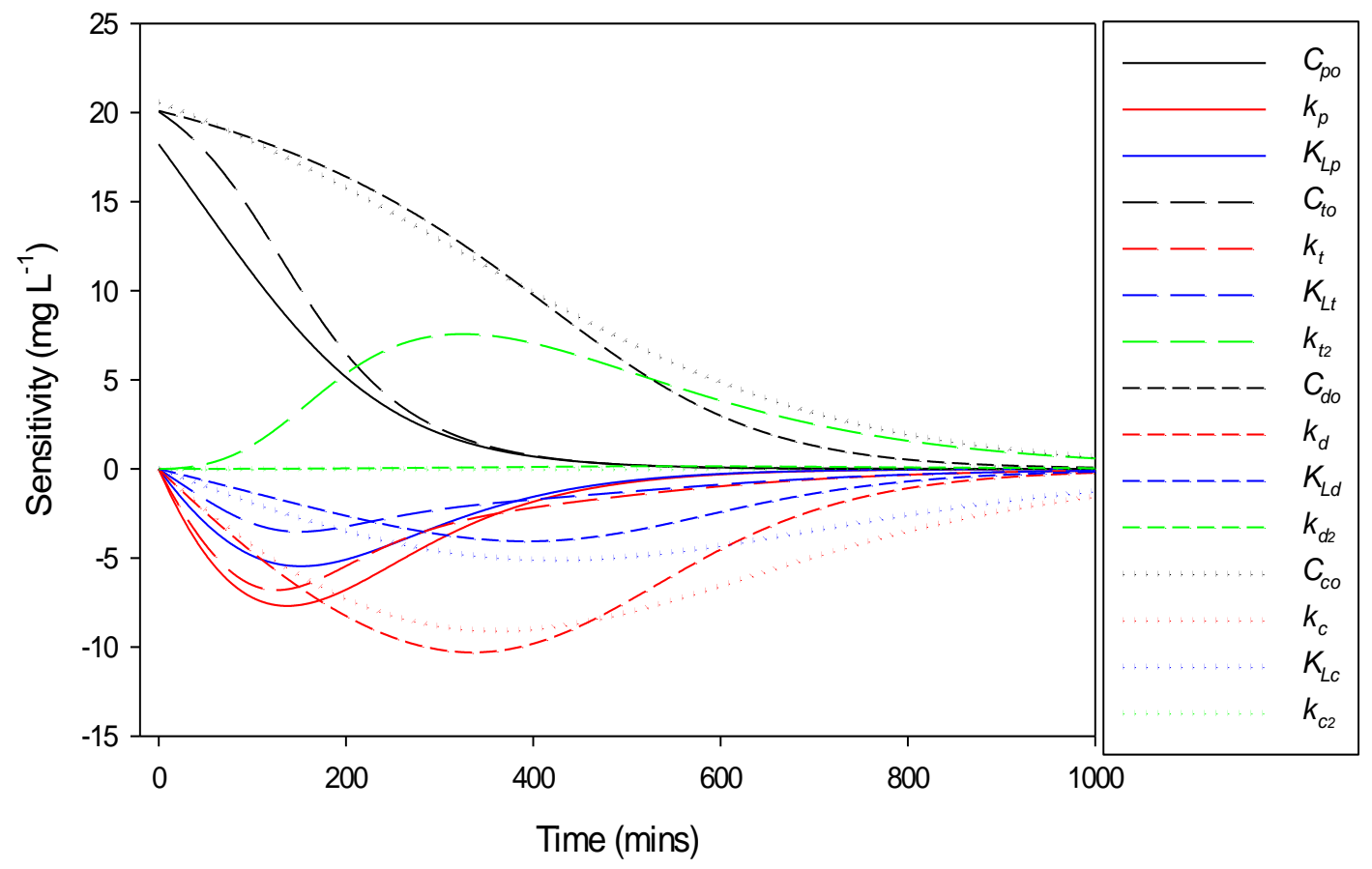

Fig.5.4.1. Time course of the sensitivity functions of $C_{p}, C_{t} C_{d}$ and $C_{c}$ with respect to LangmuirHinshelwood kinetics in reactor D.

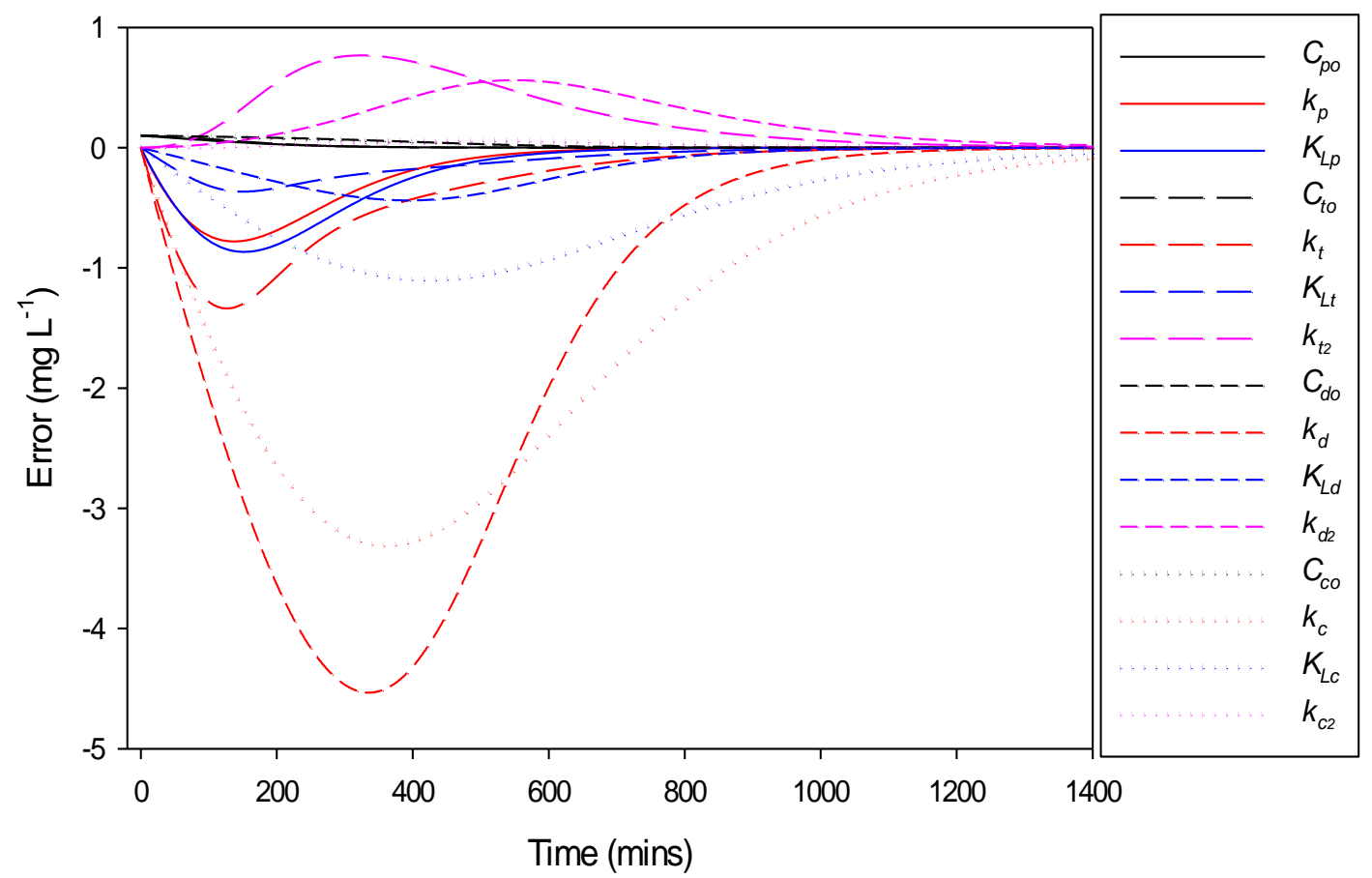

Fig.5.4.2. Error contributions of model parameters on the calculated concentrations of $C_{p}, C_{t} C_{d}$, and $C_{c}$ with respect to Langmuir-Hinshelwood kinetics in reactor $D$. 
calculated concentrations decrease with increases in $K_{L n}$ and decrease with increases in $k_{n}$. The magnitude of changes per unit $k_{n}$ is greater than those of $K_{L n}$. The rate of formation $\left(k_{n 2}\right)$ has a positive relationship with the calculated concentration. The relative response of $k_{n 2}$ however decreases with increases in $k_{n}$. Fig.5.1.2 shows estimated parameter uncertainty analyses in reactor A. Uncertainty analysis are the tools implemented for calculating and representing the certainty with which the model results represent reality. The difference between a deterministic model result and reality will arise from model parameter errors and data errors. Data errors can only result from sampling, analysis, and researcher inconsistency and calculations errors, these are assumed to be minimal. The noticeable characteristics of Fig.5.1.2 is the uncertainty range of the degradation rate constants $\left(k_{n}\right)$ and formation rate constants $\left(k_{n 2}\right)$ in comparison to the other two estimated parameters $\left(K_{L n}\right.$ and $\left.C_{n o}\right)$. The latter parameters were calculated from experimental data with given uncertainties included in the form of determined standard deviations, and are expected not to deviate noticeably from estimated parameter values. $k_{n}$ and to a greater degree $k_{n 2}$ are model estimated values derived from estimations of set standard deviations of 10 percent in the model. It was the goal to achieve, to within an acceptable tolerance, an error-reflective model by increasing the uncertainty to a reasonable limit to allow for unknown factors, as the model boundary condition errors are simulation program specific and not user defined. Figs.5.4.1 and 5.4.2 shows the sensitivity analyses in reactor $D$, which are similar to reactor $A$. The differences are particular to the photocatalysis and formation of 4-CP, reactor $D$ has a change per unit time of the $k_{c}$ better than in reactor $\mathrm{A}$, and less change per unit time for $k_{c 2}$. Figs.5.2 and 5.3 show similar sensitivity profiles, the difference in comparison to reactors $A$ and $D$ can be attributed to the efficiency of degradation and conversion of higher level substituted chloride phenols discussed.

\section{Conclusion}

Studies of adsorption equilibrium isotherms of chlorophenols on titanium dioxide were conducted. The profiles were compatible with the Langmuir expression, and linearisation calculations performed to determined parameters. Photocatalytic dechlorination of the chlorophenols was achieved under experimental conditions. The influence of the number of substituted chloride ions favoured the most substituted compounds ahead of less chloride populated compounds. The sequence order was found to follow PCP > TCP > DCP > 4-CP, though dichlorophenol and 4-chlorophenol were relatively matched in some data sets. A modified Langmuir-Hinshelwood model was proposed, kinetic parameter estimation were performed, as well as sensitivity and uncertainty analyses.

\section{Acknowledgements}

Funding from the Water Research Commission (WRC) of South Africa through the WRC Project No. K5/1717 awarded to Prof Evans M.N. Chirwa of the University of Pretoria. 


\section{Reference}

[1] S. Lathasree, A.N. Rao, B. SivaSankar, V. Sadasivam, K. Rengaraj, Heterogeneous photocatalytic mineralisation of phenols in aqueous solutions, Journal of Molecular Catalysis A: Chemical. 223 (2004) 101-105.

[2] A.P. Davis, C.P. Huang, The removal of substituted phenols by a photocatalytic oxidation process with cadmium sulfide, Water Res. 24 (1990) 543-550.

[3] S. Malato, P. Fernández-Ibáñez, M.I. Maldonado, J. Blanco, W. Gernjak, Decontamination and disinfection of water by solar photocatalysis: Recent overview and trends, Catalysis Today. 147 (2009) 1-59.

[4] Y. Ku, R. Leu, K. Lee, Decomposition of 2-chlorophenol in aqueous solution by UV irradiation with the presence of titanium dioxide, Water Res. 30 (1996) 2569-2578.

[5] S. Sharma, M. Mukhopadhyay and Z.V.P. Murthy, Separation \& Purification Reviews 42 (2013) 263-295.

[6] J. Ângelo, L. Andrade, L.M. Madeira, A. Mendes, An overview of photocatalysis phenomena applied to NOx abatement, J. Environ. Manage. 129 (2013) 522-539.

[7] M. Inagaki, F. Kang, M. Toyoda, H. Konno, Chapter 13 - Carbon Materials in Photocatalysis, in: M.I.K.T. Konno (Ed.), Advanced Materials Science and Engineering of Carbon, ButterworthHeinemann, Boston, 2014, pp. 289-311.

[8] M. Yasmina, K. Mourad, S.H. Mohammed, C. Khaoula, Treatment Heterogeneous Photocatalysis; Factors Influencing the Photocatalytic Degradation by TiO2, Energy Procedia. 50 (2014) 559-566.

[9] A.R. Ribeiro, O.C. Nunes, M.F.R. Pereira, A.M.T. Silva, An overview on the advanced oxidation processes applied for the treatment of water pollutants defined in the recently launched Directive 2013/39/EU, Environ. Int. 75 (2015) 33-51.

[10] K.G. Linden, M. Mohseni, 2.8 - Advanced Oxidation Processes: Applications in Drinking Water Treatment, in: S. Ahuja (Ed.), Comprehensive Water Quality and Purification, Elsevier, Waltham, 2014, pp. 148-172.

[11] S. Higashimoto, N. Kitao, N. Yoshida, T. Sakura, M. Azuma, H. Ohue, Y. Sakata, Selective photocatalytic oxidation of benzyl alcohol and its derivatives into corresponding aldehydes by molecular oxygen on titanium dioxide under visible light irradiation, Journal of Catalysis. 266 (2009) 279-285.

[12] C. Minero , G. Mariella , V. Maurino , and E. Pelizzetti, Langmuir 16 (2000) 2632-2641.

[13] L. Elsellami, S. Pigeot-Rémy, F. Dappozze, F. Vocanson, A. Houas, C. Guillard, Comparison of initial photocatalytic degradation pathway of aromatic and linear amino acids, Environ. Technol. 31 (2010) 1417-1422.

[14] A.M. Fox, and M.T. Dulay, Heterogeneous Photocatalysis, Chemical reviews. 93 (1993) 341341-357.

[15] R.M. Hoffmann, S.T. Martin, W. Choi, D.W. Bahnemann, Environmental applications of semiconductor photocatalysis, Chemical reviews. 95 (1995) 65-96. 
[16] M. Pera-Titus, V. Garćia-Molina, M.A. Baños, J. Giménez, S. Esplugas, Degradation of chlorophenols by means of advanced oxidation processes: a general review, Applied Catalysis B: Environmental. 47 (2004) 219-256.

[17] V. Augugliaro, M. Bellardita, V. Loddo, G. Palmisano, L. Palmisano, S. Yurdakal, Overview on oxidation mechanisms of organic compounds by TiO2 in heterogeneous photocatalysis, Journal of Photochemistry and Photobiology C: Photochemistry Reviews. 13 (2012) 224-245.

[18] O. Hamdaoui, E. Naffrechoux, Modeling of adsorption isotherms of phenol and chlorophenols onto granular activated carbon: Part I. Two-parameter models and equations allowing determination of thermodynamic parameters, J. Hazard. Mater. 147 (2007) 381-394.

[19] C.H. Giles, T.H. MacEwan, S.N. Nakhwa, D. Smith, Studies in adsorption. Part XI. A system of classification of solution adsorption isotherms, and its use in diagnosis of adsorption mechanisms and in measurements of specific surface areas of solids, J. Chem. Soc. 10 (1960) 3973-3993.

[20] Y. Song-hu, L. Xiao-hua, Comparison treatment of various chlorophenols by electro-Fenton method: relationship between chlorine content and degradation, J. Hazard. Mater. 118 (2005) 8592.

[21] J. Bandara, J.A. Mielczarski, A. Lopez, J. Kiwi, 2. Sensitized degradation of chlorophenols on iron oxides induced by visible light: Comparison with titanium oxide, Applied Catalysis B: Environmental. 34 (2001) 321-333.

[22] F.J. Benitez, J. Beltrán-Heredia, J.L. Acero, F.J. Rubio, Rate constants for the reactions of ozone with chlorophenols in aqueous solutions, J. Hazard. Mater. 79 (2000) 271-285.

[23] P. Reichert Aquasim 2.0 - User Manual, Swiss Federal Institute for Environmental Science and technology. (1998) CH 8600 Dubendorf, Switzerland, ISBN 3-906484-16-5. 